\title{
Dimeric and dipolar ground state orders in colloidal molecular crystals
}

\author{
EMMANUEL TRIZAC ${ }^{1}$, SAMIR EL SHAWISH ${ }^{2}$ and JURE DOBNIKAR ${ }^{2}$ \\ ${ }^{1}$ Université Paris-Sud, Laboratoire de Physique Théorique et Modèles Statistiques (CNRS UMR 8626), 91405 Orsay Cedex (France) \\ ${ }^{2}$ Department of Theoretical Physics, Jožef Stefan Institute, Jamova 39, 1000 Ljubljana, Slovenia \\ Manuscript received on May 2, 2008; accepted for publication on April 1, 2009
}

\begin{abstract}
A two dimensional colloidal suspension subject to a periodic substrate evolves into a colloidal molecular crystal under situations of strong confinement. We focus on the long range orientational order thereby emerging, in the ground state. We study by simulations the situations where in each trap lies a pair of identical colloids, or alternatively a pair of oppositely charged macroions. We consider square or triangular geometries for the periodic confinement, together with less symmetric distorted lattices.
\end{abstract}

Key words: colloidal molecular crystals, confined colloidal suspensions, orientational ordering, screened Coulomb interactions, simulated annealing.

\section{INTRODUCTION AND BACKGROUND}

Whereas Coulombic interactions are recognized as essential to the understanding of the phase and structural properties of colloidal suspensions in the broad sense, there has been comparatively little work devoted to the behaviour of charged composite objects in a solution. The spherical shape is, however, more the exception than the rule in the colloidal realm, and to illustrate the nonintuitive features of the coupling between anisotropy of a macroion charge distribution (a colloid), and screening by an atmosphere of microions, we consider the simple dumbbell problem of two identical spherical colloids of charge $q$ in an electrolyte of Debye length $\kappa^{-1}$ (the solvent is hereafter considered as a structure-less medium of constant dielectric permittivity). One may naively think that, at a large distance from the above dimer, one recovers an isotropic (screened) electrostatic potential $\phi$, as is the case in vacuum where only the monopo-

Selected paper presented at the IUTAM Symposium on Swelling and Shrinking of Porous Materials: From Colloid Science to Poromechanics - August 06-10 2007, LNCC/MCT.

Correspondence to: Emmanuel Trizac

E-mail: trizac@lptms.u-psud.fr lar contribution matters. To appreciate why such an expectation is incorrect, we resort to Debye-Hückel theory (see e.g. Levin 2002, Levin et al. 2003) where $\phi$ obeys $\nabla^{2} \phi=\kappa^{2} \phi$. Denoting $2 d$ the distance between the two colloids and $\psi$ the angle between the colloids' centerto-center line and the vector (with modulus $r$ ) joining the dimer middle to an arbitrary point where the potential $\phi$ is computed (see Fig. 1), we can write $\phi$ as the superposition of two screened Coulomb potentials, which admits the large $r$ form

$$
\phi(r, \psi) \sim q \frac{e^{-\kappa r}}{r} \cosh (\kappa d \cos \psi) .
$$

Remarkably, the angular and radial dependencies factorize, so that the anisotropy of the source of the potential is relevant at all scales, at variance with vacuum or plain dielectric phenomenology. Another interesting characteristics emerges when one considers a dipole (i.e. a doublet $+q /-q$, with again inter-center distance $2 d$, see Fig. 5): $\phi$ may again be written as the sum of two screened Coulomb contributions, which now differ by the sign of their prefactors, so that again for large distances:

$$
\phi(r, \psi) \sim q \frac{e^{-\kappa r}}{r} \sinh (\kappa d \cos \psi) .
$$


This expression is of the same order in $r$ as Eq. (1), and more generally, all multipoles contribute to the leading term in the large distance decay of the electric potential for an arbitrary charge distribution (Trizac et al. 2002, Agra et al. 2004a, Ramirez and Kjellander 2006).

Surprisingly, these considerations are essential to understand the phase behaviour of the recently obtained colloidal molecular crystals, where a light lattice of traps produced by interfering laser beams induces the crystallization of an otherwise two dimensional suspension of spherical colloids. Such systems exhibit a complex phase diagram that has been studied experimentally (Brunner and Bechinger 2002), numerically and theoretically (Reichhardt and Olson 2002, Agra et al. 2004b, Reichhardt and Olson-Reichhardt 2005, Sarlah et al. 2005, 2007, El Shawish et al. 2008). For illustrative purposes, we consider the case of a rectangular lattice of confining traps in a strong pinning regime where there are exactly two colloids per trap, thereby forming a dimer. Restricting for simplicity the analysis to nearest neighbor interactions, and assuming that the large distance form (1) holds, we see that the potential created by a single dimer is minimum in the $\psi=\pi / 2$ direction, so that a pair of interacting dimers minimizes its repulsion in the parallel configuration, when both are perpendicular to the line joining their centers (shown with the double arrow of length $l$ in Fig. 1). Such a pair configuration does not allow to construct a trivial ground state on the square lattice, and leads to a frustrated situation. We have, therefore, resorted to numerical simulations to analyse the corresponding order that arises. On the other hand, the situation appears simpler when dipoles are considered on the square lattice: from Eq. (2) a given pair of dipoles maximizes its attraction when both are aligned to their center-to-center separation. A plausible ground state then naturally emerges, with stripes of aligned dipoles with alternating orientation (up and down) from stripe to stripe. In such a configuration, not all pairs of dipoles are in the optimal configuration though, due to the lattice geometry. In this case, and to analyse other lattice geometries, numerical simulations are necessary to clarify the orientational ordering that is selected by the interactions considered. It is essential here to realise and keep in mind that the energy of a given configuration is not invariant upon si- multaneously rotating all dimers or dipoles orientations, at variance with Heisenberg spins.

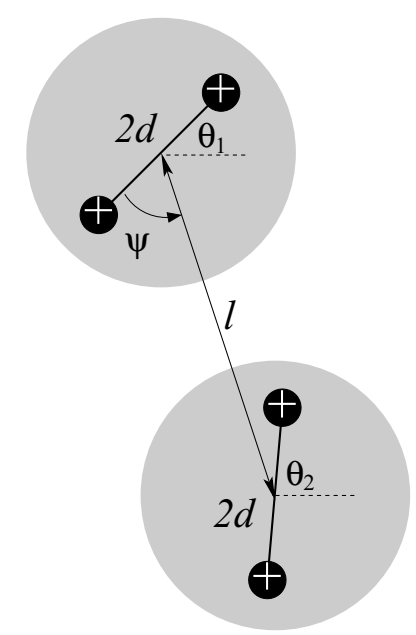

Fig. 1 - Schematic view of charged dimers in two different traps (shaded circles). $l$ is the distance between the neighboring traps, $2 d$ is the size of the "colloidal molecule" and $\theta_{1,2}$ are the characteristic angles. The large distance potential created by the upper dimer in the direction of the second one is given by Eq. (1), where the angle $\psi$ is shown on the figure.

In the following, we will concentrate on the ground state of the system, with the idea that the orderings observed experimentally at strong pinning amplitudes correspond to a regime where thermal agitation effects become irrelevant. We shall address both dimer and dipole cases, on rectangular and triangular lattices of light traps. We assume that the (2D) traps are isotropic, with no preferred direction. Such a point of view differs from that adopted in (Sarlah et al. 2005, 2007), and corresponds to a different pinning regime, see (El Shawish et al. 2008) for a discussion. In section 2 where we consider dimers, we will discuss ground state phase diagrams and be in particular concerned with the relevance of envisioning a dimer in a trap as a rigid object. In the case of dipoles (section 3), such an assumption is more natural due to the strong attraction between two oppositely charged colloids confined in the same trap, and we will, therefore, consider the dipole as a bound entity, which allows for a reduction of the complexity of the problem. The paper is partly based on (El Shawish et al. 2008), where the dipolar case was not addressed though. 

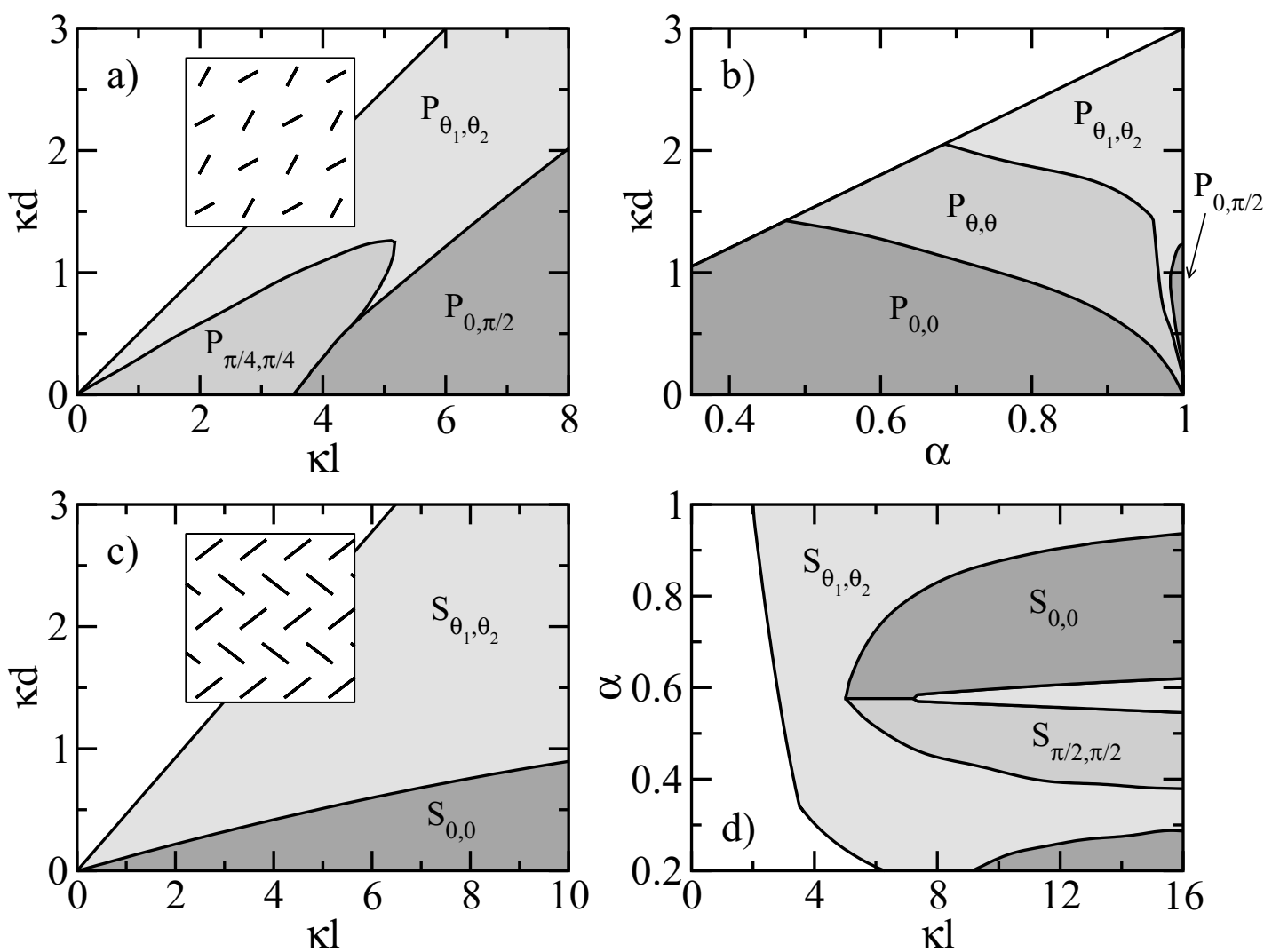

Fig. 2 - Summary of phase diagrams for rigid dimers on a rectangular lattice (upper row) for a) $\alpha=1$ and b) $\kappa l=6$, and on a triangular lattice (lower row) for c) $\alpha=0.9$ and d) $\kappa d=1$. Colours denote the different phases with particular values of the bipartite angles $\theta_{1}$ and $\theta_{2}$. Note that the void (white) regions correspond to unphysical parameter ranges such as $d \leq \alpha l / 2$ (a given trap cannot extend further than half the inter trap distance).

\section{REPULSIVE IN-TRAP INTERACTIONS}

Here we consider the dimeric case with two colloids of the same charge per trap. We distinguish between a simplified model of trapping where a fixed dimer size is assumed (rigid dimer model), and the "full" problem where the dimer is allowed to adjust its size to the confining potential imposed. This leads in some cases to a spontaneous symmetry breaking where all dimers do not have exactly the same size in neighboring wells.

In the rigid approach, the intra-trap colloidal distance is fixed to a value $2 d$ (see Fig. 1) and we consider that the rigid dimers only have a rotational degree of freedom $\left(\theta_{i}\right.$ in Fig. 1). The ground state is then determined by minimizing the screened Coulombic energy:

$$
E_{c}=K \sum_{i \neq j} \frac{\exp \left(-\kappa r_{i j}\right)}{\kappa r_{i j}}
$$

where $K$ is here immaterial since the focus in on ground state properties, $\kappa$ measures the range of the screened interaction, and $r_{i j}$ denotes the distance between colloids $i$ and $j$ (the sum above runs over all possible pairs of colloids). The trapping potential is not accounted for, but implicitly taken into account through the $d$ value: stronger confinement leads to a decrease of this distance. Within such an approach, the relevant dimensionless parameters are $\kappa d$ and $\kappa l$ (see Fig. 1). In addition, we introduced the aspect ratio $\alpha$ (rescaling all distances in one principal direction of the lattice by a factor $\alpha$ ). For instance, if $\alpha=1$ corresponds to a square geometry, $\alpha \neq 1$ is then for a rectangular unit cell.

Minimizing the Coulombic energy with a simulated annealing method, we obtained the different phases mentioned in Figure 2. These phases are bipartite, characterized by two angles $\theta_{1}$ and $\theta_{2}$ : checkerboard-like on 

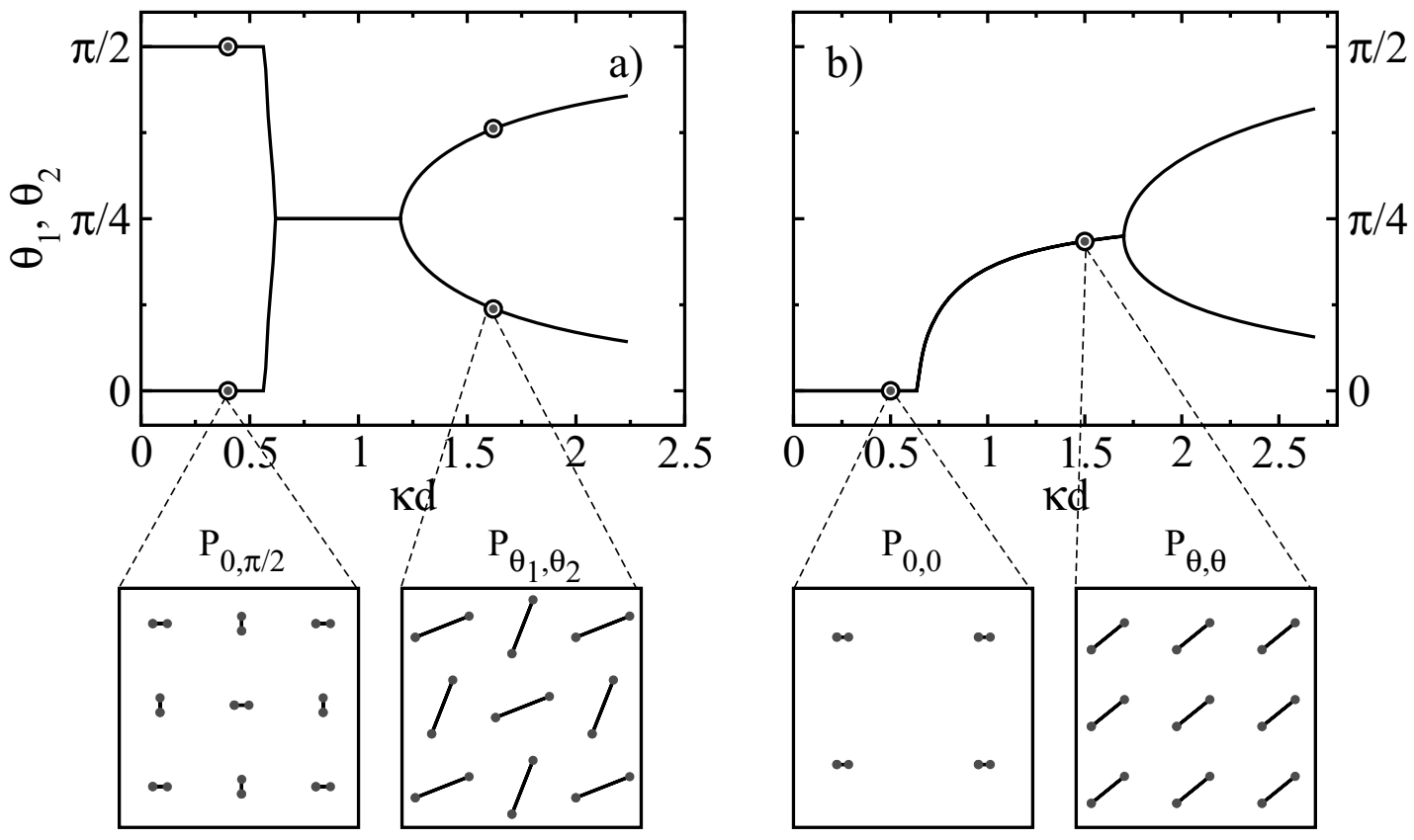

Fig. 3 - Comparison between the ground states for dimers on a square substrate, obtained considering the full "flexible" model including explicitly the trapping potential (results shown with circles), against the restricted approach where the dimers are considered as rigid objects (results shown by the continuous curves). (a) $\kappa l=4.5$, $\alpha=1$ and (b) $\kappa l=6, \alpha=0.9$. The insets display the typical configurations in both rigid and flexible models.

the square lattice (see the inset in the upper row), and in the form of parallel stripes on the triangular lattice. We name these orders $P_{\theta_{1}, \theta_{2}}$ and $S_{\theta_{1}, \theta_{2}}$ respectively. In particular, the $P_{0, \pi / 2}$ phase corresponds to an order reminiscent of an antiferromagnetic phase (with alternating "vertical" and "horizontal" dimers on neighboring traps). It should be emphasized that a phase indexed by an unspecified angle $\theta$ or a pair of angles $\theta_{1}, \theta_{2}$ exhibits an order that can be tuned upon changing the parameters $(\alpha, \kappa d, \kappa l)$. An example showing how characteristic angles change is provided in Figure 3. On the other hand, there exists other phases where the angles are constant throughout the whole domain of existence, see e.g. phases $P_{\pi / 4, \pi / 4}$ in Figure 2-a) or $S_{0,0}$ and $S_{\pi / 2, \pi / 2}$ in Figure 2-d). We note that, on the equilateral triangular lattice, phases $S_{0,0}$ and $S_{\pi / 2, \pi / 2}$ coincide, but this is no longer the case in the distorted situation where $\alpha \neq 1$.

Although a priori natural, the rigid dimer assumption significantly restricts the phase space of the system and may prevent the formation of more exotic phases than those reported above. To explore the corresponding possible shortcomings, we have relaxed the assumption of a fixed distance $d$ between the colloids, taking thereby due account of the confining potential. We considered that the two colloids in a given trap suffer a harmonic potential with a minimum at the trap center. Modifying the relative importance of harmonic confinement versus Coulomb repulsion, the mean intra-trap colloid distance can be tuned. For a meaningful comparison of the rigid and flexible scenarios, the mean colloid distance $2 d$ is measured in the full "flexible" approach, and then used in a rigid model simulation. Figure 3 shows that on the square lattice, both routes lead to the same results. However, on the triangular lattice, we have observed that a tetrapartite ordering may emerge at large enough $\kappa d$, see Figure 4-a). For moderate values of $\kappa d$, we observed a good agreement rigid/flexible, see e.g. Figure 4-b) which corresponds to a vertical cut in Figure 2d), with a reentrant $S_{\theta_{1}, \theta_{2}}$ phase as the aspect ratio increases: starting from $S_{\theta_{1}, \theta_{2}}$ at low $\alpha$, the system evolves continuously into a $S_{\pi / 2, \pi / 2}$ phase, changes then ab- 

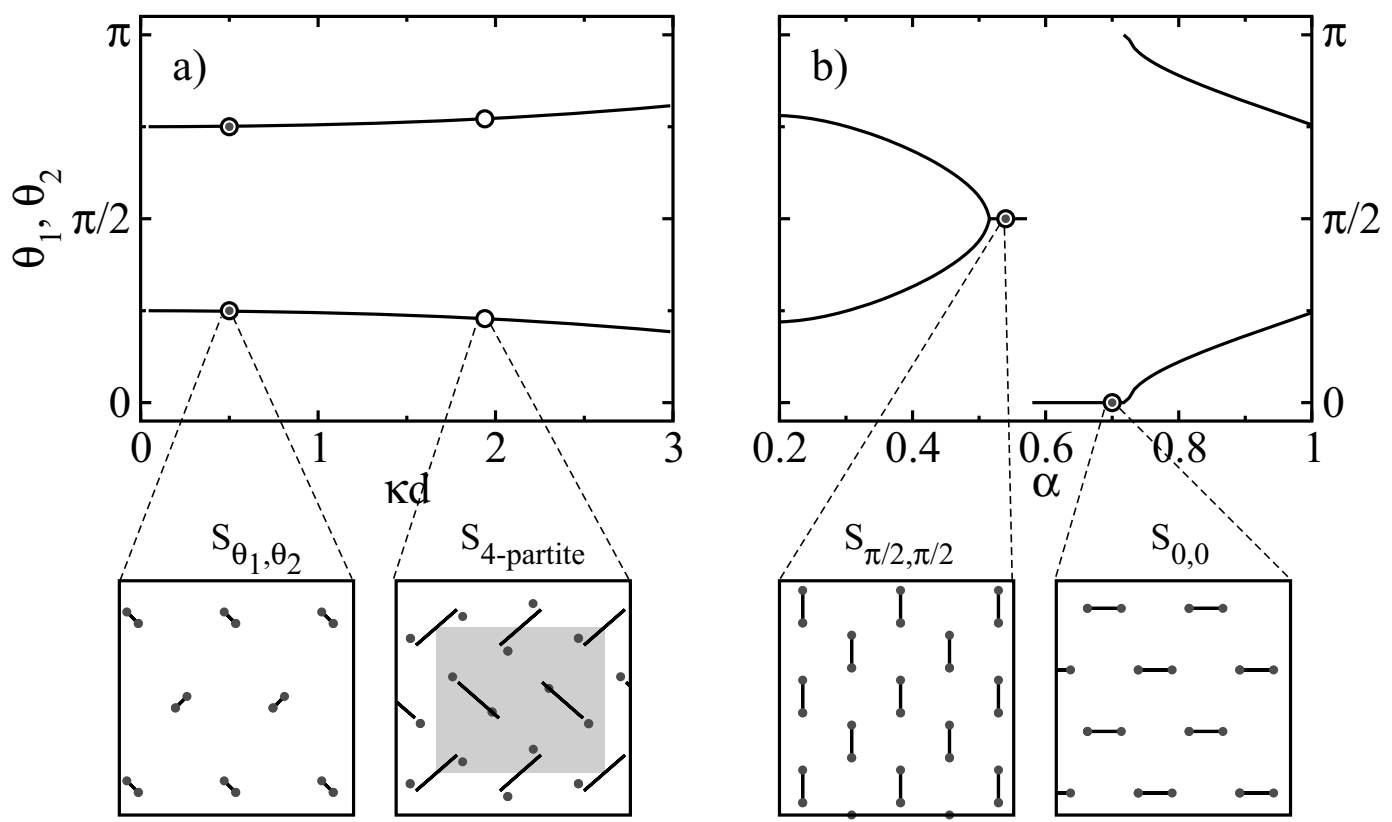

Fig. 4 - Same as Figure 3 but on a triangular lattice with (a) $\kappa l=6, \alpha=1$ and (b) $\kappa l=6, \kappa d=1$. In the equilateral configuration ( $\alpha=1)$, tetrapartite ground state configurations appear for flexible dimers with large $\kappa d$ (shaded region in a)). A typical tetrapartite configuration (disks) with enlarged unit cell is shown in the inset along with the "rigid" bipartite configuration (lines) for comparison.

ruptly to an $S_{0,0}$ and is finally back to the $S_{\theta_{1}, \theta_{2}}$, with, however, more separated characteristic angles (larger value of $\left.\left|\theta_{1}-\theta_{2}\right|\right)$, than in the low $\alpha$ regime.

\section{ATTRACTIVE IN-TRAP INTERACTIONS}

Our interest now goes to the dipolar case, with two oppositely charged colloids per trap (see Fig. 5). As alluded to earlier, it becomes irrelevant to distinguish between the rigid and flexible cases: due to the strong colloidal attraction, the dipole behaves as a rigid object. As might have been anticipated, the ground state on the rectangular lattice is of $P_{-\pi / 2, \pi / 2}$ fashion, see Figure 6 . The numerical results have again been obtained with simulated annealing. However, when the relevant structures have been identified, with the correct sublattices, the problem at hand depends on a small number of parameters, and lends itself to a straightforward direct energy minimization. We have compared both approaches (annealing and direct minimization), that give very similar results.

On the other hand, the phase diagram for triangular lattices is more diverse, with additional antiferro-

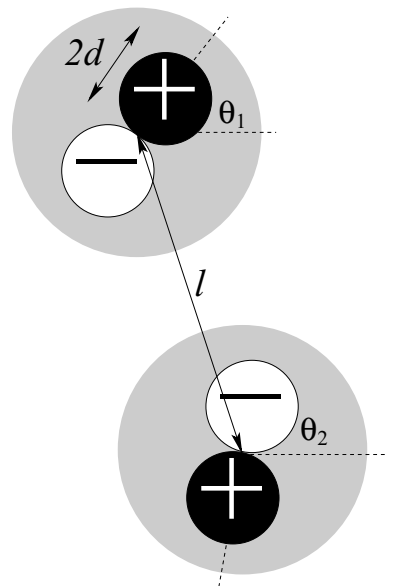

Fig. 5 - Schematic representation of two electric dipoles, i.e. dimers composed of positively and negatively charged colloids, in the traps (shaded circles). Here $2 d$ is the size of a colloid whereas the other parameters have the same meaning as in Figure 1.

magnetic $S_{\theta, \pi+\theta}$ and ferromagnetic $S_{\theta, \theta}$ stripe phases, as shown in Figure 7. It can be seen again that small parameter changes may trigger large orientational rearrangements that may be abrupt. We also note that the $S_{\theta, \pi+\theta}$ phase reported in Figure 7-b), and sketched in 

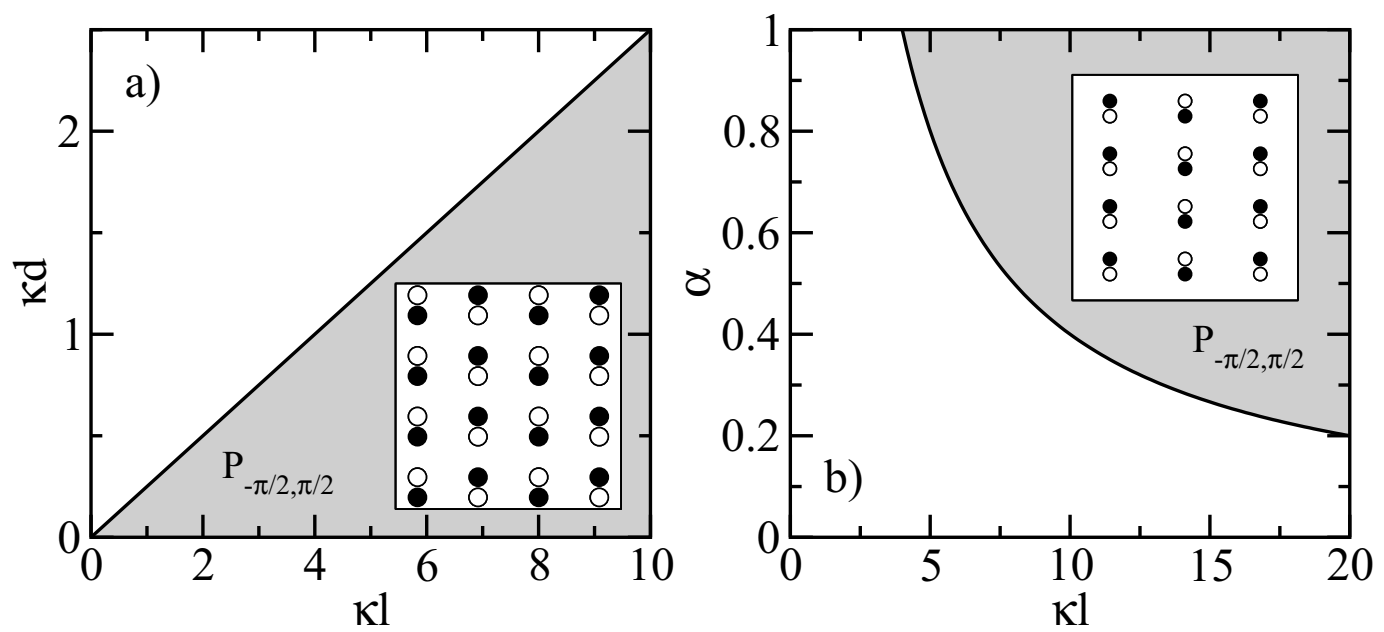

Fig. 6 - Ground state phase diagram of oppositely charged dimers on a rectangular lattice for (a) $\alpha=1$ and (b) $\kappa d=1$. The $P_{-\pi / 2, \pi / 2}$ phase sketched in the insets is the ground state for all values of $\alpha, \kappa l$ and $\kappa d$.
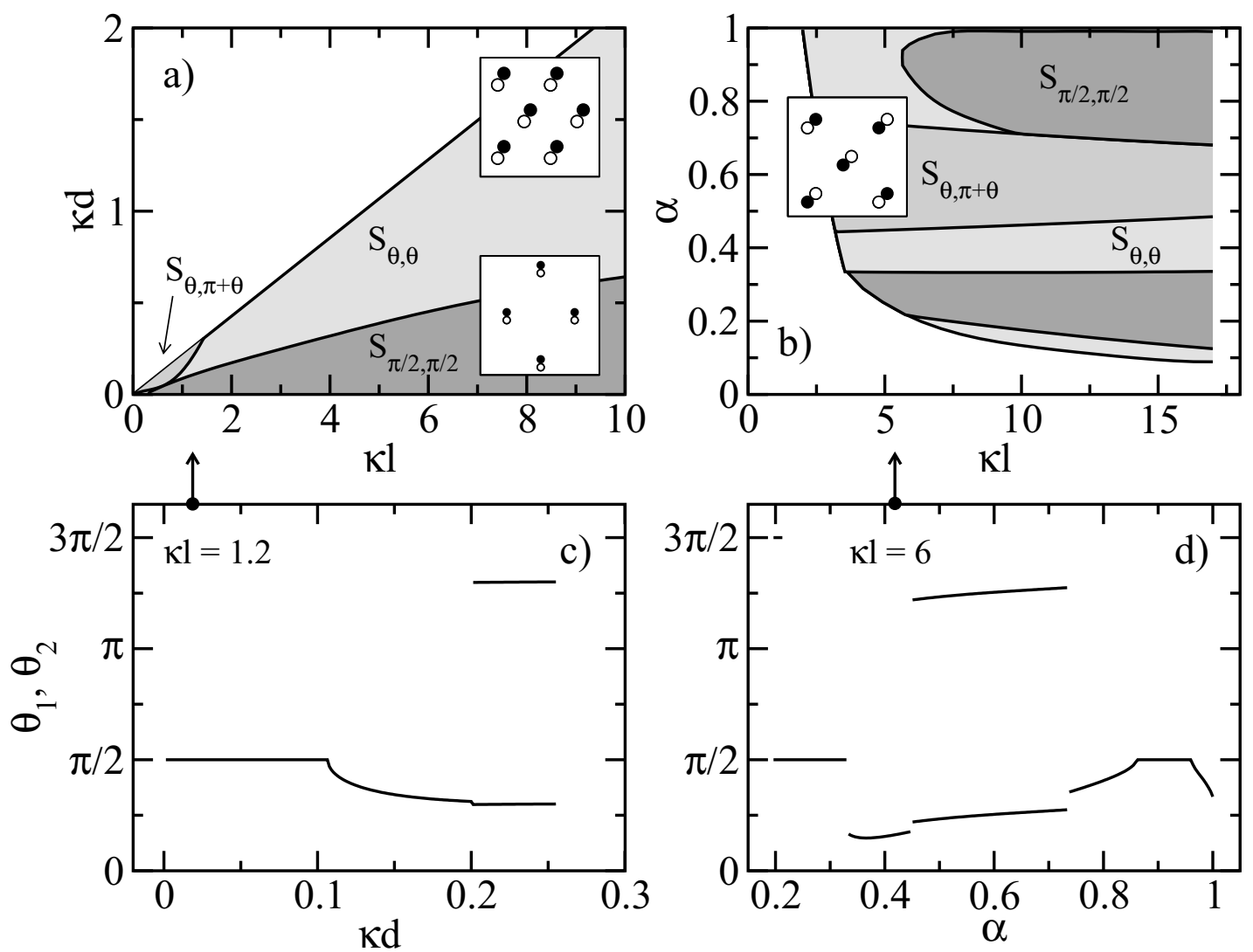

Fig. 7 - Phase diagram for opposite charged dimers on a triangular lattice for (a) $\alpha=0.8$ and (b) $\kappa d=0.5$. The insets show the representative stripe configurations. The parameter dependence of bipartite angles along the $\kappa l=1.2$ and $\kappa l=6$ lines of phase diagrams (a) and (b) is shown in (c) and (d), respectively. 

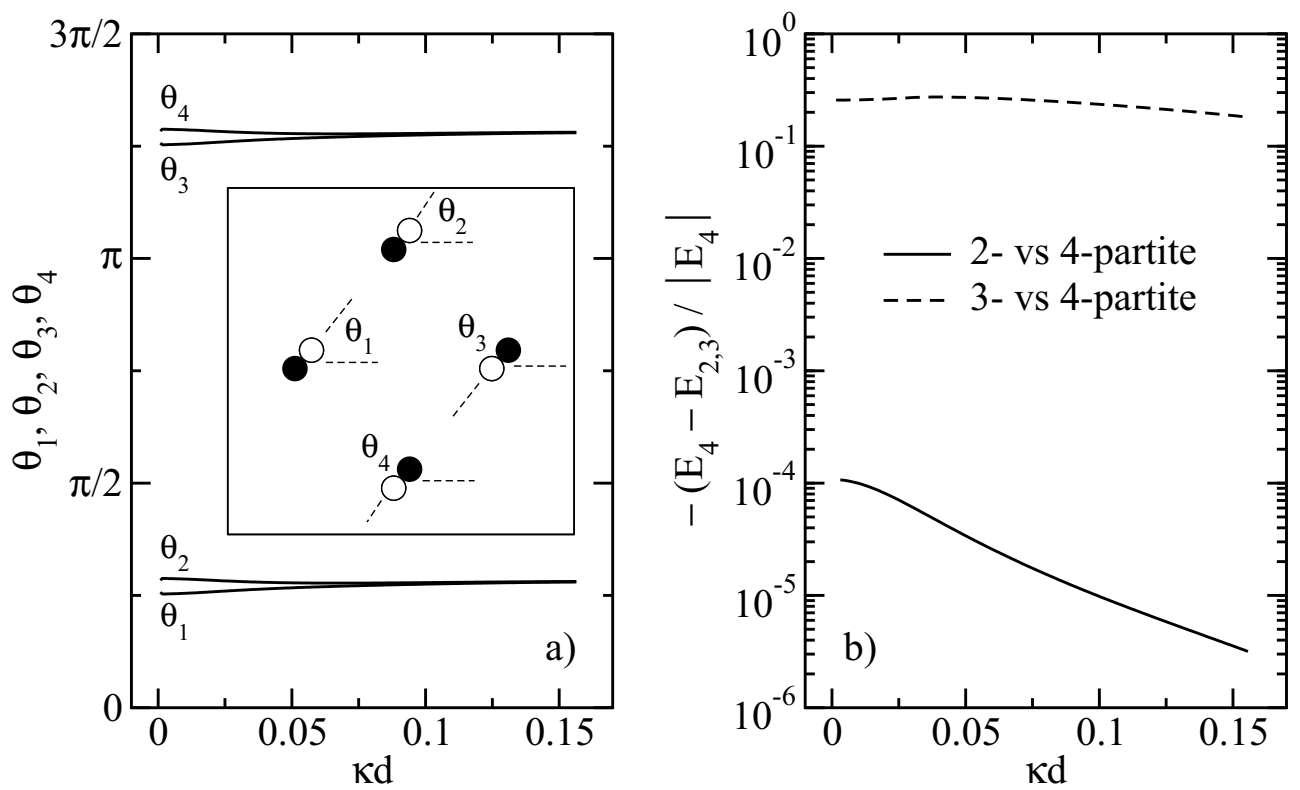

Fig. 8 - Emergence of tetrapartite ground state configurations for opposite charged dimers on a triangular lattice with $\alpha=0.7$ and $\kappa l=0.8$ : (a) the parameter dependence of tetrapartite angles and (b) a comparison of ground state energies $E_{i}$ calculated for bipartite $\left(E_{2}\right)$, tripartite $\left(E_{3}\right)$ and tetrapartite $\left(E_{4}\right)$ lattices. In the inset of (a) we show the tetrapartite unit cell configuration for $\kappa d=0.05$.

the inset, is reminiscent of the ground state $P_{-\pi / 2, \pi / 2}$ found on the square lattice, which is itself a stripe phase. The reason for this similarity is clear: with the particular scaling factor $\alpha=1 / \sqrt{3} \simeq 0.57$, the triangular lattice is mapped onto a square one (with principal axis rotated by an angle of $\pi / 4$ with respect to the horizontal direction corresponding to a principal axis of the unscaled original triangular lattice). For $\alpha \simeq 0.57$ we therefore expect a $S_{\pi / 4,5 \pi / 4}$ phase on the triangular lattice. This is precisely what is observed in Figure 7-d) where one can see that the couple $\left(\theta_{1}, \theta_{2}\right)$ hits the value $(\pi / 4,5 \pi / 4)$ at $\alpha=1 / \sqrt{3}$ (see also the inset of Fig. 7-b) for a visual confirmation). Finally, in the low screening regime and for $\alpha<1$, we have observed a tendency towards tetrapartite ordering, as shown in Figure 8. This tendency is, however, weak: the resulting angles only slightly differ from those in the bipartite structure (it can be seen in graph 8-a) that $\left.\left|\theta_{2}-\theta_{1}\right| \ll \theta_{1}\right)$ and, furthermore, the energy differences involved are faint (Fig. 8-b)).

\section{CONCLUSION}

We have found by numerical simulations the orientational orders associated to the ground state of the so- called colloidal molecular crystals, that are obtained experimentally when a two dimensional colloidal liquid of highly charged colloids is subject to a modulated light lattice. The forces arising from light pressure and from the dielectric mismatch between the solvent and colloids tend to confine the colloids in the regions of largest laser intensity. We investigated the cases of a square and triangular symmetry for the resulting periodic confining potential, together with distorted geometries obtained by applying a scaling factor $\alpha$ to say the $y$ coordinate, leaving the $x$ coordinate unaffected. We have addressed two different situations, where either two like-charge colloids are present in every trap (dimeric case) or where the two colloids are oppositely charged. In the latter case, we restricted ourselves to cases where the resulting bound object is of vanishing charge (referred to as the dipolar case). The orientational orderings obtained are rich and display a variety of phases. Most of them are associated to a bipartite lattice, with stripe or checkerboard arrangements. A weak tendency to tetrapartite ordering has been reported, which, however, does not alter the qualitative features of the predictions obtained enforcing bipartition of the lattice. 
While the dimeric case has been realised experimentally, we are not aware of any experimental studies with oppositely charged colloids. A first difficulty to overcome lies in the fact that, starting from a weakly modulated situation and gradually increasing confinement amplitude, the system may not organize spontaneously with exactly one dipole per trap. On the other hand, with dimers, the strong colloid-colloid repulsion ensures that there are no defects in the corresponding crystal, i.e. exactly one dimer per trap.

Finally, we emphasize that the Coulombic energy considered here [Eq. (3)] considers all pairs of dimers/ dipoles in the system, and does not assume that only interactions between nearest neighbor traps are relevant. The nearest neighbor assumption might seem natural at first glance, given the exponential dependence with respect to distance of the screened Coulomb potential. It may, however, prove incorrect, as has been uncovered in (El Shawish et al. 2008).

\section{ACKNOWLEDGMENTS}

We would like to thank Frédéric van Wijland and A. Sarlah for useful discussions. The work was supported by the Slovenian Research Agency through grants P1-0055 and Z1-9303. We acknowledge the support of the bilateral program Proteus supported by the Slovenian Research Agency and the french Ministère des Affaires Etrangères et Européennes.

\section{RESUMO}

Uma suspensão bidimensional coloidal sujeita a um substrato periódico evolui para um cristal coloidal molecular em situações de forte confinamento. Nós focamos na ordem de orientação emergindo a partir do estado fundamental. Fazendo uso de simulações, estudamos as situações onde em cada armadilha reside um par de colóides idênticos ou, alternativamente, um par de macro-íons de cargas opostas. Consideramos geometrias quadradas ou triangulares para o confinamento periódico com arranjos simétricos com menor distorção.

Palavras-chave: cristais moleculares coloidais, suspensões coloidais confinadas, ordenamento de orientação, interações de Coulomb, recozimento simulado.

\section{REFERENCES}

Agra R, Trizac E AND Bocquet L. 2004a. The interplay between screening properties and colloid anisotropy: towards a reliable pair potential for disc-like charged particles. Eur Phys J E 15: 345-357.

Agra R, VAN WiJland F AND TRIZAC E. 2004b. Theory of Orientational Ordering in Colloidal Molecular Crystals. Phys Rev Lett 93: 018304.

BRunNer M AND Bechinger C. 2002. Phase Behavior of Colloidal Molecular Crystals on Triangular Light Lattices. Phys Rev Lett 88: 248302.

El Shawish S, Dobnikar J AND TrizaC E. 2008. Ground states of colloidal molecular crystals on periodic substrates. Soft Matter 4: 1491.

LEVIN Y. 2002. Electrostatic correlations: from Plasma to Biology. Rept Prog Phys 65: 1577-1632.

LEVIN Y, TRIZAC E AND BocQuet L. 2003, On the fluidfluid phase separation in charge stabilized colloidal suspensions. J Phys Condens Matt 15: S3523-S3536.

RAMIREZ R AND KJellander R. 2006. Effective multipoles and Yukawa electrostatics in dressed molecule theory. J Chem Phys 125: 114110.

ReICHhardt C. AND Olson CJ. 2002. Novel Colloidal Crystalline States on Two-Dimensional Periodic Substrates. Phys Rev Lett 88: 248301.

ReICHHARDT C AND Olson-REICHHARdt CJ. 2005. Ordering and melting in colloidal molecular crystal mixtures. Phys Rev E 71: 062403.

Sarlah A, Franosch T And Frey E. 2005. Melting of Colloidal Molecular Crystals on Triangular Lattices. Phys Rev Lett 95: 088302.

Sarlah A, Frey E And Franosch T. 2007. Spin models for orientational ordering of colloidal molecular crystals. Phys Rev E 75: 021402.

Trizac E, Bocquet L, Agra R, Weis JJ And Aubouy M. 2002. Effective interactions and phase behaviour for a model clay suspension in an electrolyte. J Phys Condens Matt 14: 9339-9352. 